\title{
RAPID GAS FUELING IN A BARRED POTENTIAL BY SELF-GRAVITATIONAL INSTABILITY
}

\author{
Keiichi WADA and Asao HABE \\ Department of Physics, Hokkaido University, Sappro 060 Japan
}

Recent IRAS surveys have revealed that large infrared luminosities are originated from active star forming regions in galaxies (Soifer et al. 1986). Such starburst regions are frequently located in galactic central regions and $\mathrm{CO}$ observations indicate that these regions contain a large amount of molecular gas (e.g. Ishizuki et al. 1990). However, the triggering mechanism for starbursts and the mechanism of the high mass supply rate of gas into a galactic center are still unclear.

It is suggested that tidal encounters of galaxies remove angular momentum of gas and trigger rapid gas accretion and starburst. Noguchi(1988) has shown by computer simulations that galaxy-galaxy interactions induce a stellar bar, and gas loses its angular momentum and accumulates to a galactic center. In his numerical simulation, non-axisymmetric potential of a stellar bar plays an important role in the accretion of gas. However, it is not obvious as to whether or not gas accretes into a nuclear region within a few hundred pc only by the effects of stellar bar.

There were a number of studies concerning gas dynamics in rotating bar potential to find the mechanism which forms and maintains galactic spiral arms (e.g. Matsuda et al. 1987). These numerical studies revealed that oval gas rings are formed near the two $I L R$. The rings are generally elongated and the major radius of the ring nearly coincides with the radius at outer $I L R$. Although a large fraction of gas inside the $\mathrm{CR}$ radius is accumulated into the ring, gas cannot accrete to the galactic center beyond inner $I L R$. However, self-gravity of gas is not taken into account in these simulations.

If a pattern speed of the weak barred potential induced by galaxy-galaxy encounters, which has suitable for $I L R$ s, some fraction of gas in the galaxy is rapidly accumulated into the near proximity of $I L R$ radius. In this case, if self-gravity of the accumulated gas is taken into account, the gas becomes gravitationally unstable and it is expected that a rapid gas supply into the center of the galaxy would occur.

We investigate the dynamics of self-gravitating gas in the weak barred potential by 2-D numerical simulation, and show that for the initial gas mass ratio to stellar mass grater than $10 \%$, a central elongated gas ring becomes unstable and collapses after gas accumulates to form the elongated 
gas ring near the $I L R$ radius(Wada and Habe 1992). To analyze this rapid and complex process, we made a video of this simulation. The video clearly shows that the formation of dense gas clumps at both sides of the elongated ring triggeres instability of the ring: The dense clumps move and collide with each other on a highly elongated orbit; After frequent collisions, these gas clumps merge and finally form a central dense core. Instead of the video, we show here the time evolution of the velocity of gas in the ring (Fig.1).

\section{References}

Ishizuki, S. et al. 1990. Nature, 344,224.

Matsuda, T. et al. 1987. Mon. Not. R. astr. Soc., 229, 295.

Noguchi,M.,1988. Astr. Astrophys., $203,259$.

Soifer, B. T.,1984. Astrophys. J., 283,L1.

Wada,K. \& Habe,A., 1992. Mon. Not. R. astr. Soc., 258,82.
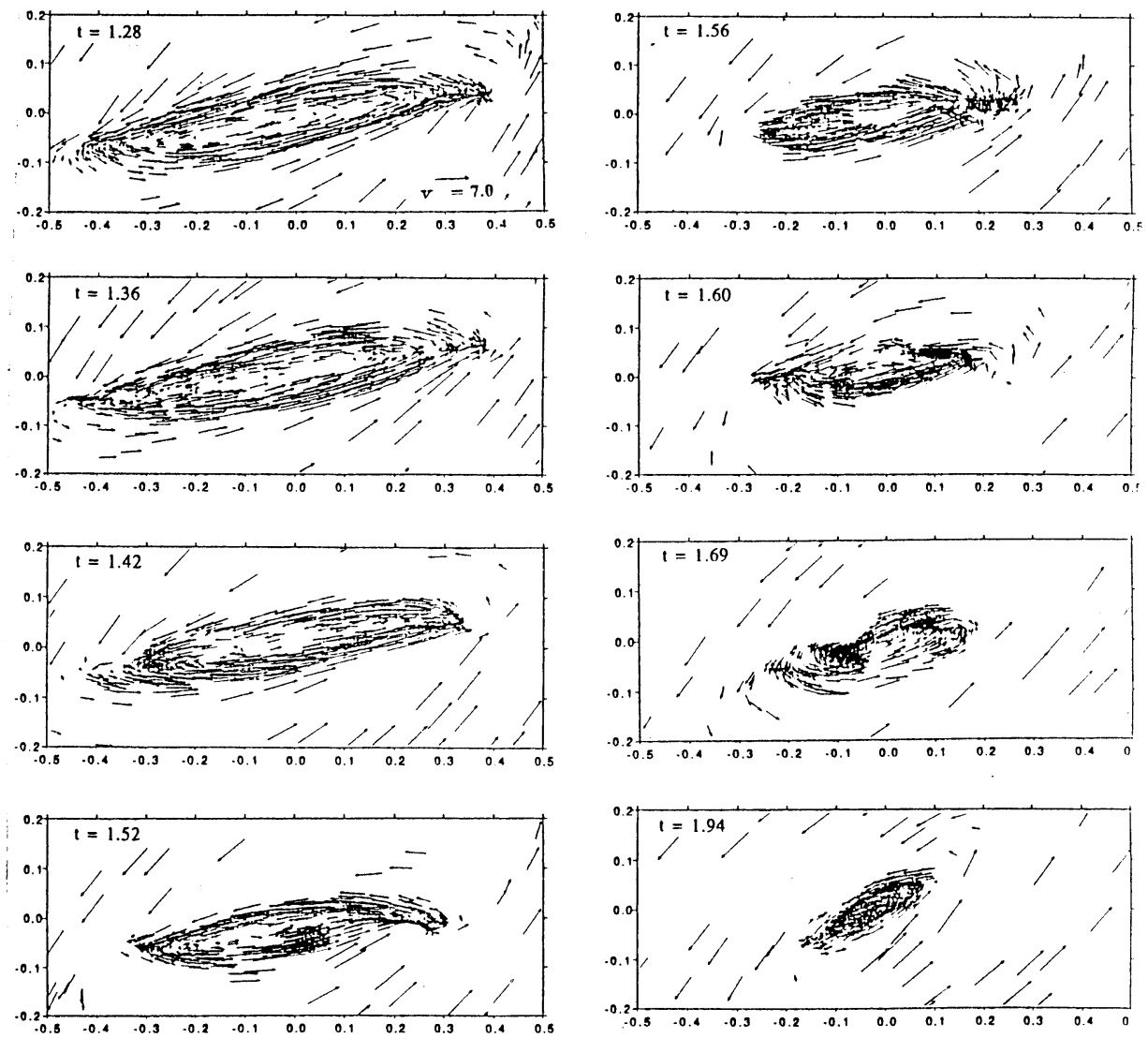

Figure 1: Collapse of the gas ring. The arows represent the velocity vector of the gas. The unit of the time is $3.5 \times 10^{8} \mathrm{yr}$. 